\title{
Perfiles de los profesionales de la información: entre lo tradicional y lo emergente ${ }^{*}$
}

\author{
Johann Pirela Morillo \\ Ph. D. en Ciencias Humanas, doctor \\ en Bibliotecología y Estudios de la \\ Información, magíster en Educación, \\ magíster en Museología, licenciado en \\ Bibliotecología y Archivística. Docente \\ e investigador adscrito al Departamento \\ de Estudios de la Información de la \\ Universidad de La Salle, \\ Bogotá-Colombia. \\ jepirela@unisalle.edu.co \\ http://orcid.org/0000-0002-1492-6053
}

\section{Marisol Salazar Álvarez}

Maestranda en Ciencia de la Información. Bibliotecóloga. Profesora de cátedra en la Escuela Interamericana de Bibliotecología de la Universidad de Antioquia,

Medellin-Colombia. marisol.salazar@udea.edu.co https://orcid.org/0000-0003-2358-2587

\begin{abstract}
Resumen
En este artículo se exponen los resultados de la investigación "Caracterización de los egresados de Archivística, Bibliotecología y Ciencia de la Información en Colombia: 2013-2018" a partir de los elementos de formación, reconocimiento profesional y mercado laboral, para determinar la correspondencia entre la formación profesional y las dinámicas del campo laboral, el impacto del egresado, la pertinencia social y curricular. Estos resultados posibilitan la identificación de necesidades y retos para cualificar la formación profesional, consolidar la pertinencia y fortalecer el impacto de los egresados en el campo laboral; además, se destaca su vigencia respecto a estudios anteriores: alta empleabilidad y baja movilidad; bibliotecas universitarias y archivos administrativos de carácter estatal como principal empleador; los sectores educación y servicios como principales fuentes de empleo, con predominancia del contrato a término definido como modalidad contractual y con remuneración de entre 2 y 3 salarios mínimos legales mensuales vigentes. Estos resultados están en sintonía con los actuales contenidos de formación, especialmente desde la perspectiva de los conocimientos fundamentales, y en baja sintonía con áreas emergentes como documentación electrónica, blockchain, analítica de datos, ciencia abierta, big data e inteligencia de negocios; conocimientos que se enmarcan en la Revolución 4.0 y en los retos que deben enfrentar estos profesionales en correspondencia con otros del campo de la información y del conocimiento: las fake news, el fenómeno de la desinformación y la manipulación a la que se somete la publicación de la información en redes sociales.
\end{abstract}

Palabras clave: perfil profesional, perfil laboral, archivística, bibliotecología, ciencia de la información, innovación curricular.

Cómo citar este artículo: Pirela, Johann; Salazar, Marisol (2021). Perfiles de los profesionales de la información: entre lo tradicional y lo emergente. Revista Interamericana de Bibliotecología, 44(3), e344766. https://doi.org/10.17533/udea.rib. v44n3e344766

Recibido: 2021-04-19 / Aceptado: 2021-08-30

* Artículo derivado de la investigación "Caracterización de los egresados de Archivística, Bibliotecología y Ciencia de la Información en Colombia: 2013-2018”. Proyecto de investigación adscrito al Centro de Investigaciones en Ciencia de la Información (CICINF) de la Escuela Interamericana de Bibliotecología de la Universidad de Antioquia y desarrollado en convenio con la Universidad de la Salle. Investigadora principal: Orlanda Jaramillo, coinvestigadores: Johann Pirela Morillo, Dorys Liliana Henao Henao y Marisol Salazar Álvarez. 


\section{Profile of Information Professionals: Between the Traditional and the Emerging}

\begin{abstract}
The article sets out the results of the research "Characterization of graduates of Archival Science, Library Science, and Information Science in Colombia: 2013-2018" based on the recognition of the professional in the labor field and the correspondence between training and needs of the environment; key elements for the evaluation and decision-making in the curricular processes and the design of policies that strengthen the relationship with the working environment. The results of this research made it possible to identify current needs and challenges to qualify professional training processes, to consolidate the relevance of academic programs, and to strengthen the impact of graduates in the labor field. It is highlighted how the results of previous studies persist or are valid: High employability and low mobility; university libraries and state administrative archives as the main employer; the education and services sectors as the main sources of employment, with a defined term contract employment relationship as the predominant contractual modality and with remuneration of between 2 and 3 current legal monthly minimum wages. These results are in medium tune with current training content, especially from the perspective of fundamental knowledge, and in low tune with emerging areas such as electronic documentation, blockchain, data analytics, open science, big data, and business intelligence. All this is framed in Revolution 4.0 and in the problems that these professionals must face in correspondence with other problems in the field of information and knowledge, such as fake news, the phenomenon of disinformation, and the manipulation of the information on social networks.
\end{abstract}

Keywords: Professional profile, work profile, archival science, library science, information science, curricular innovation.

\section{Introducción}

Los constantes cambios en los escenarios laborales demandan permanentes replanteamientos y modificaciones en los procesos formativos, tanto por parte de las instituciones de educación superior (IES) como de los programas de formación. Replanteamientos que están orientados hacia la alta calidad para atender los retos planteados por el desarrollo científico y tecnológico y la producción de bienes y servicios, en convergencia con los propósitos del desarrollo económico, social, político y cultural en armonía con el medio ambiente del país; especialmente la vinculación con los objetivos de desarrollo sostenible (ODS) y los desafíos planteados por la crisis social y económica generada en el mundo por la COVID-19. Tales retos se corresponden con las funciones sustantivas de la educación superior: docencia, investigación y extensión-proyección social.

En Colombia, se inicia la formación profesional en el campo de la archivística, la bibliotecología y la ciencia de la información en 1956 con el programa de Bibliotecología ofrecido por la Escuela Interamericana de Bibliotecología (EIB) de la Universidad de Antioquia, Medellín. Posteriormente, surgieron propuestas de formación profesional en el área: la Universidad de La Salle (1971, Bogotá) con el programa Sistemas de Información y Documentación; la Pontificia Universidad Javeriana (1973, Bogotá) con el programa Ciencia de la información - Bibliotecología; la Universidad del Quindío (1986, Armenia) con Ciencia de la Información y la Documentación, Bibliotecología y Archivística; la Fundación Universitaria UNINPAHU (2001, Bogotá) con Profesional en Ciencia de la Información; la Universidad Industrial de Santander (UIS) (2013, Bucaramanga) con Historia y Archivística; y, más recientemente, la Universidad Distrital Francisco José de Caldas (2018, Bogotá) con el programa en Archivística y Gestión de la Información Digital. Otras universidades también han ofertado formación en el área, en diferentes momentos históricos, como es el caso de la Universidad Nacional de Colombia (1965, Bogotá) y la Universidad de La Costa (2012, Barranquilla). Además de programas técnicos y tecnológicos en diferentes instituciones en el país. En este sentido, es importante mencionar, por ejemplo, los programas ofrecidos por el Sena en Gestión Bibliotecaria y Gestión Documental, que aún están vigentes; programas que manifiestan la necesidad de reflexionar sobre sus procesos curriculares, su correspondencia y diálogo con las tendencias globales de la evolución de conceptos relacionados con el área de formación - como son dato, información, conocimiento, memoria y TIC - , con las habilidades blandas, con los principios y posturas pedagógicas y con la fundamentación epis- 
temológica que da el sustento teórico a la formación de los profesionales de la información. ${ }^{1}$

Es así como algunas de las universidades que actualmente ofrecen formación profesional (Javeriana, La Salle, Universidad de Antioquia y UNINPAHU) han introducido recientemente cambios en el currículo, entre los que se destacan las revisiones sobre los aspectos que están marcando un desarrollo en el tratamiento, organización y difusión de la información y la documentación. Es el caso de la preservación digital, los contextos de posverdad y las fake news, las metrías de la ciencia, la vigilancia tecnológica y la Revolución 4.0. Esta última reconocida por expertos como un nueva etapa del desarrollo productivo e industrial, también llamada la industria inteligente, que plantea la integración de diversas aplicaciones y herramientas tecnológicas, como la realidad virtual y aumentada, el internet de las cosas, la ciencia de datos, cloud-computing, la seguridad de la información, entre otros. Asuntos que demandan procesos formativos mucho más interdisciplinares frente al trabajo de los profesionales de la información en los diferentes escenarios sociales, económicos, políticos y educativos en los que se desempeñen y que convocan a reconfigurar el alcance, los fines y los procesos de gestión históricamente atribuidos a los archivos, bibliotecas, centros de documentación y a todo tipo de unidad de información, como mediadores y certificadores de la validez y veracidad de la información.

Ante estas realidades y demandas emergentes, como la actual crisis mundial generada por la COVID-19 y la virtualidad en casi todas las acciones humanas derivadas de esta situación, las IES y los programas de formación profesional en el área están llamadas a realizar ejercicios de evaluación, de revisión sistemática de las tendencias y ajustes curriculares, de manera permanente, para garantizar la correspondencia de las ofertas formativas con las necesidades planteadas por los sectores socioproductivos y por las demandas más apremiantes del desarrollo sostenible; para cuya realización, la gestión de la información, la documentación y el conocimiento constituyen procesos transversalmente estratégicos. Desde estas nuevas demandas de formación, surge la necesidad de caracterizar al egre-

1 El término profesionales de la información hace alusión a archivistas, bibliotecólogos y profesionales en ciencia de la información. sado de los programas de Archivística, Bibliotecología y Ciencia de la Información en Colombia; por lo tanto, esta investigación se convierte en un documento que puede apoyar los mejoramientos curriculares, pues los procesos de transformación e innovación curricular requieren de información actualizada sobre lo que está demandando el mercado laboral, los cambios de perfil, las características, el impacto del profesional al medio laboral y la pertinencia social de los profesionales.

Desde esta perspectiva, se planteó la realización de la investigación "Caracterización de los egresados de Archivística, Bibliotecología y Ciencia de la Información en Colombia: 2013-2018"; investigación que mediante el enfoque mixto, las técnicas de búsqueda documental, grupo focal, small data y encuesta buscó caracterizar a los egresados desde el análisis de los datos relacionados con los aspectos socioeconómicos, laborales (ubicación, cargo, salario, contratación, funciones), de formación profesional, de reconocimiento social y de expectativas profesionales (Jaramillo, Henao, Pirela \& Salazar, 2020). Aspectos que están alineados con las tendencias organizacionales, en cuanto a la gestión documental, la organización de la información y, también, con las tendencias pedagógicas, académicas y normativas en la educación superior, específicamente en el campo de acción de los profesionales de la información. Para tal efecto, se determinó como criterio geográfico y temporal seleccionar a los egresados de los programas de Bibliotecología y Archivística de la EIB de la Universidad de Antioquia, Medellín; del programa Sistemas de Información, Bibliotecología y Archivística de la Universidad de La Salle; del programa Ciencia de la información - Bibliotecología de la Pontificia Universidad Javeriana; del programa Ciencia de la Información y la Documentación, Bibliotecología y Archivística de la Universidad del Quindío; y del programa Profesional en Ciencia de la Información de la Fundación Universitaria UNINPAHU. Todos obtuvieron su título profesional entre el 2013 y el 2018, en Colombia. ${ }^{2}$

2 El proceso investigativo no incluyó a los egresados del programa de Historia y Archivística de la UIS (el programa inició en el año 2013 con una duración de 10 semestres) ni a los del programa en Archivística y Gestión de la Información Digital de la Universidad Distrital Francisco José de Caldas (el programa inició en el año 2019 con una duración de 10 semestres), por no contar con egresados o contar con pocos egresados según la temporalidad definida para la investigación. 
En esta línea, en los procesos de caracterización de egresados y de evaluación de la formación profesional, es imprescindible la voz del egresado y del empleador, por ser estos actores centrales y sobre los que recae todo el proceso formativo; además, su condición de agentes externos al proceso formativo les permite una visión más objetiva de dichos procesos; por lo tanto, pueden hacer aportes más ecuánimes. Así, en el proceso investigativo participaron 224 egresados de los diferentes programas que existen en el país, de los cuales, 170 cuestionarios fueron validados por cumplir la temporalidad definida en la investigación. Estos egresados ocupan diversidad de cargos, entre ellos, directivos, coordinadores, profesionales universitarios y, en algunos casos, técnicos, tecnólogos y auxiliares (al momento de la aplicación de la encuesta a los egresados). También se contó con la participación de 17 empleadores de Medellín y Bogotá, ciudades de mayor empleabilidad en el área, y de 7 administradores académicos (decanos, directores/ coordinadores) de programas de formación profesional en el país. La información obtenida de los grupos focales permitió analizar la relación entre el perfil profesional y el perfil laboral.

En este artículo se presentan entonces los resultados de la investigación, con base en la metodología empleada en el desarrollo del proceso. A partir del análisis descriptivo y la aplicación de técnicas mixtas, se caracterizó a los egresados de archivística, bibliotecología y ciencia de la información; se propusieron nuevos escenarios de desempeño profesional y la pertinencia de los profesionales de estas áreas como insumo tanto para los procesos de transformación e innovación curricular, que requieren de información actualizada sobre las demandas del mercado laboral, como también para continuar procesos de seguimiento a egresados. Estos procesos deben contribuir a mejorar y cualificar la oferta formativa y, además, facilitarles a los egresados el acceso a mejores oportunidades laborales. Los resultados dan cuenta de datos generales de los egresados, tomados de la encuesta aplicada: condiciones socioeconómicas, desempeño profesional, satisfacción laboral y formación profesional. Además de los datos anteriores, también se presentan análisis desde el desarrollo de los grupos focales y desde ofertas laborales publicadas en portales web.

\section{Metodología}

La investigación tuvo como punto de partida el estudio exploratorio, analítico e interpretativo de la información relacionada con los egresados de archivística, bibliotecología y ciencia de la información de los programas profesionales existentes en Colombia al año 2019; lo que implicó un análisis cualitativo y cuantitativo de los datos recuperados en diferentes fuentes de información, con el fin de dar respuesta al objetivo: "Caracterizar el egresado de los programas de archivística, bibliotecología y ciencia de la información en el contexto laboral colombiano, graduados entre el año 2013-2018, para determinar la pertinencia de los planes de estudio" (Jaramillo et al., 2020). De esta manera, el proceso de investigación se realizó desde un enfoque mixto, que argumenta su uso por la verificación de los datos (cuantitativos y cualitativos), la comprensión integral del problema y la credibilidad de los datos: "la integración sistemática de los métodos cualitativo y cuantitativo en un solo estudio con el fin de obtener una fotografía más completa del fenómeno" (Hernández-Sampieri, Fernández-Collado \& Baptista-Lucio, 2014, p. 534); esto posibilitó la mejor comprensión de lo investigado. Fue un enfoque combinado con el método diseño exploratorio secuencial (DEXPLOS), que permitió la combinación de técnicas e instrumentos de recolección de información y precisó la elaboración de una matriz de análisis para la interpretación de la información. Con este método se hizo una exploración inicial del problema investigado, que implicó las siguientes fases derivativas: 1) recolección de información y extracción de datos (búsqueda documental y de datos de portales de empleo); 2) análisis con enfoque cualitativo de los resultados obtenidos en la fase $1 ; 3$ ) diseño, aplicación y análisis de la encuesta; 4) diseño, realización de grupos focales y análisis de información; 5) integración de los resultados cualitativos y cuantitativos, a partir de los cuales se dio respuesta a los objetivos específicos:

- Identificar el perfil profesional y laboral de los archivistas, bibliotecólogos y profesionales en ciencia de la información, egresados de los programas de formación profesional en Colombia.

- Analizar el perfil profesional y laboral de los archivistas, bibliotecólogos y profesionales en ciencia de 
la información, egresados de los programas de formación profesional en Colombia.

- Determinar la pertinencia de la formación profesional de los archivistas, bibliotecólogos y profesionales en ciencia de la información en el contexto laboral colombiano.

Atendiendo a los objetivos, el alcance investigativo fue de corte descriptivo y analítico, con el fin de especificar las propiedades y los componentes del objeto de estudio, es decir, tanto la caracterización de los egresados de archivística, bibliotecología y ciencia de la información como la descripción de las tendencias en el área. El proceso comprendió tres etapas, las cuales no fueron lineales, sino que se fueron combinando a medida que el proceso lo requería. Así, la primera etapa correspondió a la búsqueda y análisis de información cualitativa, buscó la recolección de información documental y la extracción de datos de los portales de empleo. La información recolectada en esta primera etapa permitió la construcción de los referentes teóricos, con lo cual se indagó en investigaciones desarrolladas en el último quinquenio, entre las que se destacan las de Jaramillo (2014); Ortiz-Pérez (2014); García (2014); Pirela y Pulido (2015); Pirela, Prieto y Pulido (2017); Jaramillo, Betancur y Marín (2017); Jaramillo, Betancur y Sáenz (2018); Ávila y Panesso (2018), con el fin de conocer las perspectivas sobre la caracterización de los egresados y sus aportes al mejoramiento de la calidad de los planes de estudios y con ellos de los programas. Estos datos posibilitaron el diseño de las categorías conceptuales y matrices de análisis que permitieron construir el instrumento cuantitativo (encuesta) y obtener los datos relacionados con las categorías definidas para caracterizar al egresado: socioeconómicas, de desempeño y satisfacción laboral, de valoración de la formación, de actividades de actualización y de perspectivas profesionales. En la búsqueda documental se emplearon tres tipos de fuentes: 1) bases de datos académicas -DialNet, DOAJ, Emerald ${ }^{\circledR}$, Ebsco, Redalyc, SciELO, Science Direct, Web of Science-; 2) portales de empleo LinkedIn, SIMO, el Empleo.com-; y 3) el sitio web del Observatorio Laboral para la Educación del Ministerio de Educación de Colombia.

Cabe destacar que, como parte del análisis de las ofertas de empleo disponibles en los portales de empleo mencionados, se realizaron búsquedas que se filtraron según aspectos esenciales como la denominación de los cargos, nombres de las funciones y sus descripciones, así como también las titulaciones que con mayor predominio aparecen en tales recursos. En este sentido, el criterio en el que se basó la decisión de considerar los portales de empleo fue la necesaria exploración de la perspectiva de los empleadores sobre cómo se enuncian los campos de archivística, bibliotecología y ciencia de la información.

Esta etapa incluyó la información obtenida luego de la aplicación de la técnica de grupo focal, que es una técnica de complementariedad, ya que a partir de unos datos encontrados y de su análisis e interpretación se llegó a obtener resultados más compatibles, conciliables y complementarios. La información obtenida permitió el análisis de la realidad entre pares y desde posiciones diferentes; buscando superar la subjetividad de los datos (Martínez-Miguélez, 2004). Así, la búsqueda documental permitió conocer tanto los trabajos que han abordado el tema de egresados en el contexto colombiano como los nuevos campos de formación y perfiles de los profesionales de la información. En el marco de esta investigación se realizaron dos grupos focales. Uno de ellos con empleadores de Bogotá y Medellín, por ser las ciudades donde se concentran las instituciones que forman a los egresados y por ser las de mayor empleabilidad. No fue un grupo natural de conversación ni un foro público, pues su representatividad estuvo determinada por las relaciones académicas y laborales con el tema investigado. Los directores, decanos y coordinadores de programas de formación profesional (administradores académicos) hicieron parte del segundo grupo. La información obtenida de los grupos focales permitió la mirada del egresado desde las diversas perspectivas que plantea el perfil profesional y el perfil laboral; perspectivas que solo pueden ofrecer diferentes actores (empleadores y directivos responsables de formación de programas profesionales) con variadas experiencias, intereses y valores, para tener una visión amplia, consensuada y fundamentada sobre el egresado.

La segunda etapa consistió en la búsqueda y análisis de información cuantitativa, para lo cual se diseñó y aplicó en línea, mediante un formulario de Google, la encuesta a los egresados de archivística, bibliotecología y ciencia de la información de los programas de formación pro- 
fesional de las diferentes instituciones de educación superior del país, durante los meses de marzo y abril del 2020. La difusión se realizó mediante la estrategia bola de nieve en cadena, la cual permitió que por el voz a voz y por redes sociales fueran identificados egresados clave, quienes colaboraron compartiendo la encuesta con sus contactos. En total se recibieron 224 encuestas, de las cuales se validaron 170 , luego de aplicar el filtro de año de graduación en la temporalidad 2013-2018.

La tasa de retorno no fue la esperada, debido a los cambios generados por la COVID-19 (confinamiento social) que han modificado las dinámicas de las personas, la familia, las instituciones y en sí el funcionamiento de la sociedad; cambios que justo se iniciaron en el momento de la aplicación de la encuesta, que no se pudo postergar debido a los compromisos asumidos según el cronograma. En este sentido, el equipo de investigación considera que las respuestas obtenidas representan el universo de los graduados en el periodo determinado por la investigación.

Finalmente, la tercera etapa comprendió el análisis e interpretación de resultados globales de la investigación desde una orientación cualitativa y cuantitativa, cuyo registro se realizó con el apoyo de una bitácora de análisis, en la que se consignaron conceptos, categorías y tendencias que surgieron en el proceso de análisis. Para ello se emplearon programas de análisis de datos como Excel, Tableau y Voyant (herramienta de análisis textual). Igualmente, para el análisis de la información recolectada de los grupos focales se establecieron categorías de acuerdo con áreas de formación de los profesionales de la información, para luego triangular estos datos con los obtenidos en las encuestas, en la búsqueda documental y en las ofertas laborales. Con respecto al análisis de estas ofertas (publicadas en los portales SIMO, Elempleo, LinkedIn), este se hizo mediante la metodología de small data, la cual se basa en la búsqueda de datos específicos y no en suposiciones: datos útiles, de valor, concretos, los adecuados para relacionar rápidamente los objetivos establecidos; es decir, tomar aquellos datos que aclaren el objeto investigado desde el análisis de los patrones en pequeñas muestras que advierten sobre las grandes tendencias (Lindstrom, 2016).

\section{Resultados}

La investigación, fundamentada en métodos cualitativos y cuantitativos, posibilitó llegar a diferentes conclusiones, unas relacionadas con los perfiles de los egresados, otras, con el campo laboral y otras, con la academia.

En cuanto a la caracterización de los egresados y del campo laboral, se encontraron algunas constantes o elementos que han estado presentes en los últimos estudios o investigaciones similares, realizadas a partir de la década del noventa en el país. En los resultados de la encuesta aplicada a egresados, se validaron 170 cuestionarios (tal como se puede observar en la Figura 1), en los que se destaca que la mayoría de ellos se ubica en estratos socioeconómicos medio-bajo (2 y 3 ) y comportan un promedio de edad de entre 30-35 años, ligeramente alto en relación con otras profesiones. Una posible razón es que un porcentaje alto de los egresados tenía la condición de estudiante-trabajador, por lo que sus estudios se realizaron en un tiempo mayor al estipulado en los planes de estudio. En la Pontificia Universidad Javeriana se presentó una excepción, pues el promedio de edad fue de 25-30 años. Sin embargo, la mayor parte de los egresados inicia su experiencia laboral antes de terminar los estudios profesionales, debido a la contratación laboral durante la realización de la carrera o porque vienen del mundo laboral a profesionalizarse; así, una vez obtienen el título, la institución los deja en el cargo. No obstante, este aspecto llama la atención, pues muchos egresados entran al mundo laboral en la etapa de la adultez (después de los 26 años), en comparación con la mayoría de las profesiones, que inician su ejercicio profesional en la juventud (antes de los 25 años). Por otra parte, en la caracterización del egresado, en las disciplinas relacionadas con los profesionales de la información, continúa la preferencia del género femenino, a excepción de los profesionales del programa de la Pontificia Universidad Javeriana, en el que hay un ligero aumento de egresados del género masculino.

Del campo laboral se destaca la vinculación del egresado a entidades de naturaleza pública (lo que se puede explicar desde la legislación en el área para el manejo de la documentación tanto archivística como bibliográfica), del sector de la educación y en dos tipos de unidades de 
información: bibliotecas (universitarias y públicas) y archivos (central y de gestión).

También es reiterativa la vinculación de egresados en las dos principales ciudades: Bogotá y Medellín, y en algunas ciudades como Barranquilla, Cali y Cartagena, aunque en menor proporción. Bogotá es la de mayor empleabilidad, dato que se corresponde con el tipo de ciudad (capital) y con el número de programas que se ofertan en esta ciudad. Otro elemento que vuelve a ser constante es el rango salarial, el cual varía de acuerdo con las características de la ciudad (según su categoría), la experiencia y los títulos de posgrado.

En términos generales, se encuentra flexibilidad en la vinculación laboral, precarización del trabajo y relativa estabilidad; aspectos que están en correspondencia con el tipo de contrato laboral, pero que no afectan el nivel de satisfacción laboral, al encontrarse que el porcentaje más alto de egresados expresa estar satisfecho con el cargo que ocupa, y entre los factores que mencionan están el reconocimiento profesional, las condiciones salariales y el ambiente laboral. Otro elemento que influye en la satisfacción laboral se relaciona con la contratación. Aunque la relación laboral sea por contratación de prestación de servicios o a término definido, estos son renovados una vez se terminan y de acuerdo con las normas laborales vigentes; estos factores de satisfacción laboral tienen íntima relación con la escasa movilidad laboral que tienen los egresados.

En cuanto a los hallazgos relacionados con la academia, se reitera la pertinencia y vigencia social de estas disciplinas y la valoración positiva de los contenidos de los planes de estudio, por parte de los egresados, además del reconocimiento y posicionamiento que cada vez más ganan los profesionales de la información en las instituciones en las que se desempeñan. Paralelo a ello, los egresados advierten que las áreas de formación que más contribuyen a su desempeño laboral se relacionan con las asignaturas propias del core disciplinar, seguidas de las tecnologías; no obstante, reconocen que todas las áreas/núcleos ejes de la formación son necesarios para poder dar respuesta a las demandas laborales. En este tema se advierte que las asignaturas relacionadas con las TIC y las prácticas en escenarios reales ocuparon un lugar común en cuanto a la necesidad de incorporar, potenciar y transversalizar sus contenidos en el plan de estudio. En esta misma línea, hay un llamado a la academia para que fortalezca las habilidades investigativas y las habilidades blandas en la formación profesional.

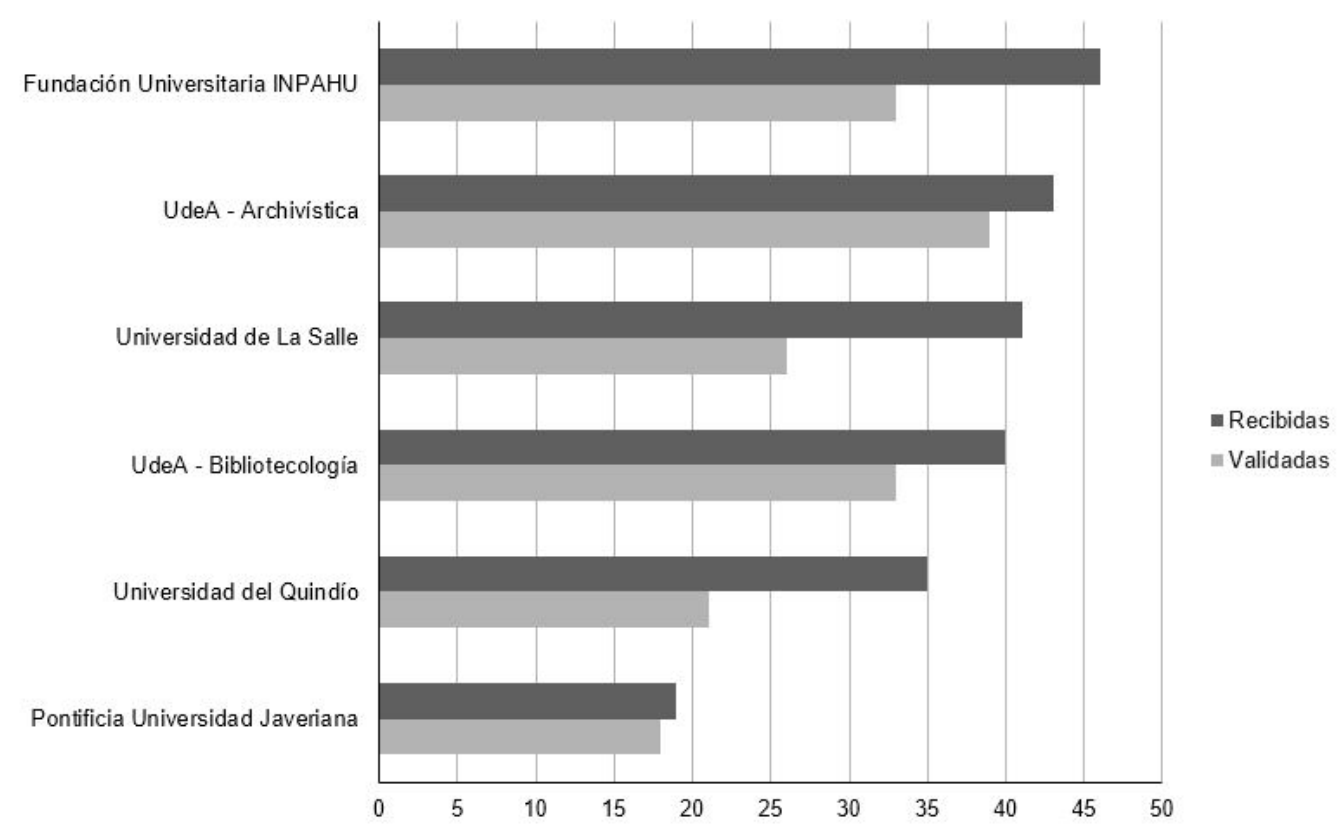

Figura 1. Resultados de la encuesta a egresados.

Fuente: elaboración propia. 
Igualmente, se identificó la importancia de conservar la impronta de cada programa en el concierto nacional $y$, en la medida de lo posible, ofertar los programas de formación con énfasis. También hay un clamor de los administradores académicos por la unificación de las denominaciones o titulaciones de la formación en $\mathrm{Co}$ lombia, de tal manera que el egresado no perciba como desacertada y engañosa la oferta formativa brindada por la universidad y, además, para que se tenga más sentido de pertenencia con la disciplina elegida. La unificación de las denominaciones junto a procesos de armonización curricular entre los programas y escuelas del país permitirá mayor movilidad entre estudiantes, en términos de agilizar convalidaciones. En relación con la unificación de criterios para la formación académica, se plantea la posibilidad de impulsar un trabajo de cooperación académica entre los programas, debido a que la propuesta curricular constituye una tecnología de conocimiento mediante la cual es posible entender la naturaleza, alcances y énfasis de la formación profesional. Esto podría ser una estrategia para identificar áreas y campos comunes, articulados desde la definición de un perfil unificado en el país, pero manteniendo la trayectoria formativa, el enfoque pedagógico y curricular que les otorga a las instituciones elementos de singularidad. Esta propuesta se articula con la necesidad de realizar las gestiones requeridas ante los entes competentes de empleo, Ministerio de Educación Nacional (Mineducación), Comisión Nacional del Servicio Civil (CNSC), para ajustar las denominaciones de las carreras y perfilar los requisitos de las ofertas y las convocatorias con las titulaciones del área, y así evitar el término amplio profesional del conocimiento en el área de las ciencias sociales y humanas.

Por otro lado, los egresados manifiestan la necesidad de insistir, en los procesos de formación universitaria, en la importancia y responsabilidad que tiene el egresado en la actualización profesional y en la continuación de estudios posgraduados, para afrontar las demandas del entorno laboral.

Entre los hallazgos relacionados con los conocimientos y habilidades emergentes, aportados del análisis de las encuestas y de los grupos focales, se destacan la incorporación en la formación profesional de temas relacionados con la gestión de documentos electrónicos y en la aplicación de los instrumentos archivísticos; tam- bién se resalta la necesidad de enfatizar en las metrías, en especial en los estudios bibliométricos. Sumado a lo anterior, está el imperativo de fortalecer las habilidades blandas: aspectos de comunicación, flexibilidad, disposición al cambio, trabajo cooperativo, creatividad, resolución de problemas, liderazgo, capacidad de negociación, entre otras. Una respuesta común en la mayoría de los egresados es el tema relacionado con la denominada industria 4.0 (internet de las cosas, blockchain, inteligencia artificial, ciencia de datos, analíticas, seguridad informática). Estos temas emergentes invitan a los programas y escuelas del país a revisar y actualizar los planes de estudio, con el fin de potenciar en la formación los aspectos relacionados con las TIC, toda vez que se identificaron oportunidades y desafíos en la transformación digital de las organizaciones.

Se identificó la posibilidad de impulsar ofertas formativas virtuales, que no solo tengan el acento en el pregrado, sino también en el nivel posgradual, en virtud de que este sería un elemento que garantiza la fidelización del egresado, con base en las fortalezas y aspectos consolidados en cada programa. Por ejemplo, en el caso de la Universidad de Antioquia, para el programa de Bibliotecología es importante continuar el trabajo sostenido en las áreas de gestión de bibliotecas públicas, alfabetización informacional, gestión del conocimiento y áreas emergentes, como es el caso de las analíticas de datos. En el caso de la Universidad de La Salle, continuar fortaleciendo la formación en el área de archivística, y en el de La Pontificia Universidad Javeriana, continuar con el impulso a la formación en el área de ciencia de datos con un perfil gerencial. La tendencia general identificada desde la caracterización de los egresados se sitúa en mantener los pregrados, pero diversificando la oferta formativa de diplomados y de maestrías, tanto en el área como enfocadas en tópicos emergentes.

A modo de síntesis, a partir de los datos de las encuestas se plantea una formación en archivística estructurada. Estas ofertas pueden movilizar acciones muy importantes de ampliación de cobertura educativa hacia los territorios, en los cuales se requiere de personal profesional en este campo. La formación bibliotecológica debe fortalecer los diálogos con aspectos de métricas de la información y del conocimiento, así como también conocimientos y habilidades pedagógicas y didácticas, 
debido a las necesidades que existen en las bibliotecas, sobre todo en las universitarias, de contar con personal con competencias para el diseño, formulación y evaluación de programas de formación de usuarios, que también incluyan componentes de la educación virtual y los criterios actuales de la educación superior, con el uso de metodologías centradas en los estudiantes, cuyo centro lo constituyen las competencias informacionales, pero vistas no solo desde una dimensión instrumental, sino también cognitiva y de aprendizaje adaptativo.

Con relación a los hallazgos del análisis de las ofertas laborales publicadas en portales como SIMO, El empleo y LinkedIn, se encontró que el número de ofertas está determinado por la naturaleza de cada portal, sea académico, público o comercial; es así como SIMO, de naturaleza pública, recoge las ofertas de los diferentes entes estatales en todo el territorio colombiano, por lo que publica un número mayor de ofertas que los portales comerciales. Una razón que puede explicar esta situación es la responsabilidad que tienen los entes estatales de cumplir las leyes relacionadas con el derecho a la información, el manejo de archivos y patrimonio y el respaldo a las tarjetas profesionales.

No obstante, el número o volumen de las ofertas no corresponde con la pertinencia de estas para los profesionales del área, debido a que los motores de búsqueda multiplican el número real de acuerdo con las palabras clave que los empleadores usen en su publicación (denominación del cargo, nombre, funciones y titulaciones). De igual forma, se encontró que, aunque un buen número de ofertas son para el área (por la descripción de las funciones o por la denominación del cargo), en las titulaciones exigidas no aparecen los títulos del área (archivística, bibliotecología y ciencia de la información); situación que es bastante determinante en el sector público, pues es el primer criterio de verificación en los postulantes. Una constante que se encontró es que en las ofertas no aparecen cargos de directivos, asesores, ejecutivos ni de especialistas que estén orientados para los profesionales del área. Se destaca que en las ofertas del sector público siempre aparecen dos requisitos: experiencia y tarjeta profesional. Los datos obtenidos de estos portales de empleo superaron los datos de las encuestas, de los grupos focales y del análisis documental (un total de 71370 ofertas, tal como puede observarse en la Tabla 1), lo que resalta la pertinencia del uso de la metodología small data, porque permitió filtrar los datos encontrados y tener resultados más precisos para el proceso de investigación.

Tabla l. Estructura para la formulación de la Estrategia Institucional CPC

\begin{tabular}{lcc}
\hline Portales & Encontradas & Seleccionadas \\
\hline Elempleo & 737 & 29 \\
LinkedIn & 2.512 & 33 \\
SIMO & 68.121 & 106 \\
Totales & 71.370 & $168(0,2 \%)$ \\
\hline
\end{tabular}

Fuente: elaboración propia.

Un reducido número de ofertas tienen los títulos de archivista, bibliotecólogo o ciencia de la información; del total de ofertas encontradas, solo el 0,2 \% especifica estos títulos. Siempre hay ofertas para otras áreas del conocimiento, especialmente para administradores e ingenieros. Bogotá, Medellín y Cali son las ciudades donde se concentra la mayor cantidad de ofertas laborales de nivel profesional, las cuales, a su vez, tienen salarios correspondientes con el nivel profesional. En ciudades intermedias o municipios del territorio colombiano, aparecen ofertas equivalentes a uno o dos salarios mínimos legales mensuales vigentes (SMLMV).

Fue reiterativo el hallazgo de muchas ofertas que tienen funciones de profesionales de la información, pero las titulaciones corresponden a otras áreas del conocimiento; en esta línea, fue común encontrar que a los cargos de secretarios de gobierno, despacho, control interno, correspondencia, catastro, de las alcaldías, se les asignen funciones de archivo (Tablas de Retención Documental [TRD], correspondencia, memoria histórica, transferencias primarias y secundarias de los documentos), en combinación con funciones de gestión de proyectos de la alcaldía; por consiguiente, son ofertas a las que aparentemente podrían aplicar los archivistas, pero por el requisito de titulación no lo pueden hacer.

Se destaca la importancia del estudio de las ofertas laborales, debido a que en ellas se especifican las funciones que está demandando el medio laboral y las posibles áreas que no estén siendo consideradas en la formación de los profesionales. De esta manera, las ofertas son una fuente de información importante para que 
la academia defina, afine o ajuste los títulos de los profesionales de la información y realice las gestiones ante las instituciones pertinentes para el reconocimiento y posicionamiento de las titulaciones del área.

Un elemento importante que cabe resaltar es la inexistencia, en el mercado laboral, de la diferenciación entre las funciones del bibliotecólogo, el archivista y el profesional en ciencia de la información; además, el hecho de que muchos cargos de los profesionales del área son para otras titulaciones, pero no a la inversa.

En cuanto al análisis de las interacciones discursivas de los participantes en los grupos focales, estos dan cuenta de nuevos horizontes que se tendrán que explorar desde los procesos de formación y plantear ofertas situadas que también coloquen su acento, además de las necesidades de las principales ciudades del país, en las características y particularidades de los territorios, en los que aún queda trabajo por hacer en términos de garantizar los cuadros profesionales para apalancar el acceso a la información, a la lectura, a la gestión de la información y del conocimiento, con perspectiva diferencial. También estos grupos focales evidenciaron la necesaria atención que se debe dar a los aspectos no solo considerados como centrales, marcados desde lo disciplinar de la bibliotecología y la archivística, sino también a las competencias blandas (lo psicoafectivo, la flexibilidad de adaptación a los cambios, la plasticidad mental, la inteligencia emocional, el liderazgo, el pensamiento crítico, creativo, analítico y sintético y la comunicación). Los empleadores advirtieron sobre la importancia de fortalecer, en la formación profesional, las prácticas en escenarios reales, la gerencia y las competencias investigativas.

\section{Conclusiones}

Se corroboró la vigencia de los resultados de estudios anteriores: alta empleabilidad y baja movilidad; las bibliotecas universitarias y los archivos administrativos de carácter estatal como principal empleador; los sectores de educación y servicios como principales fuentes de empleo; la relación laboral predominante por contratación de servicios o a término definido con salarios entre 2 y 3 SMLMV. Estos resultados están en mediana sintonía con los actuales contenidos de formación, especialmente desde la perspectiva de los conocimientos fundamentales (núcleo tradicional en la formación: "jurisdicción" o su "territorio" de formación); y en baja sintonía con áreas emergentes y las tendencias, en conexión con la triada de la gestión documental, de la información y el conocimiento y otros campos como la comunicación digital, blockchain, inteligencia de negocios, ciencia de datos, entre otros. Todo lo anterior enmarcado en la Revolución 4.0, para responder a las demandas y necesidades actuales de la sociedad, especialmente, con los fenómenos relacionados con la gestión de la información y el conocimiento, como vigilancia tecnológica, seguridad informática, preservación digital, confiabilidad de la información, fake news y la manipulación de la información tanto en las organizaciones como en redes sociales.

Se resalta la necesidad de establecer perfiles con núcleos, ejes, áreas o troncos comunes para la formación profesional y que luego se diversifiquen en la formación posgraduada, con el fin de atender las necesidades, demandas y tendencias surgidas desde los mercados laborales y los horizontes emergentes de las profesiones relacionadas con la gestión de la información, en escenarios de innovación, diversidad cultural, competitividad, productividad y creatividad, permeados por los principios expresados en la Agenda 2030 (ONU, 2015), que apuntan a la sostenibilidad.

Se hace relevante definir estrategias para generar el acercamiento constante a la realidad del entorno laboral, de los profesionales de la información, desde el estudio de las ofertas laborales y relacionar la pertinencia de los planes de formación e introducir en ellos mejoras $\mathrm{y}$ actualizaciones.

\section{Referencias}

1. Ávila, Giovanna; Panesso, Nelly (2018). Prospectiva del programa de archivística de la Universidad de Antioquía (tesis de maestría). Universidad de La Salle, Medellín. https://ciencia.lasalle.edu.co/cgi/viewcontent. cgi?article=1033\&context=maest_gestion_documental

2. García, Camilo (2014). Estudio sobre el impacto del programa académico Tecnología en Archivística en la situación laboral y en las condiciones económicas de los egresados de las seccionales de Urabá y Oriente. Universidad de Antioquia, Escuela Interamericana de Bibliotecología. 
3. Hernández-Sampieri, Roberto; Fernández-Collado, Carlos; Baptista-Lucio, María del Pilar (2014). Metodología de la Investigación (6. ed). McGraw Hill. http://observatorio.epacartagena.gov.co/wp-content/ uploads/2017/08/metodologia-de-la-investigacionsexta-edicion.compressed.pdf

4. Jaramillo, Orlanda (2014). Perfil del mercado laboral del profesional de la información y su pertinencia con la formación profesional en Colombia en el siglo XXI. Informe de investigación. Medellín Universidad de Antioquia.

5. Jaramillo, Orlanda; Betancur, María Cristina; Marín, Sebastián Alejandro (2017). La archivística como profesión: caracterización del proceso de formación de la Escuela Interamericana de Bibliotecología. Revista Interamericana de Bibliotecología, 40(3), 243-259. https://doi.org/10.17533/udea.rib.v40n3a05

6. Jaramillo, Orlanda; Betancur, María Cristina; Sáenz, Andrés (2018). Evaluación de la formación profesional en archivística: el caso de la Escuela Interamericana de Bibliotecología. Revista Interamericana de Bibliotecología, 41(3), 319-327. https://doi.org/10.17533/udea.rib.v4ln3a09

7. Jaramillo, Orlanda; Henao, Dorys; Pirela, Johann; Salazar, Marisol (2020). Informe de investigación: Caracterización de los egresados de Archivística y Bibliotecología en Colombia: 20132018. Medellín: Universidad de Antioquia.

8. Lindstrom, Martin (2016). Small data: las pequeñas pistas que nos advierten de las grandes tendencias. Grupo Planeta.

9. Martínez-Miguélez, Miguel (2004). Ciencia y arte en la metodología cualitativa. México: Trillas.

10. ONU (2015). La Agenda 2030 y los Objetivos de Desarrollo Sostenible: una oportunidad para América Latina y el Caribe. https://www.un.org/sustainabledevelopment/es/ objetivos-de-desarrollo-sostenible/

11. Ortiz-Pérez, María Isabel (2014). El perfil laboral del egresado de Tecnología en Archivística de la Universidad de Antioquia. Medellín, Universidad de Antioquia.

12. Pirela, Johann; Pulido, Nelson (2015). Perfiles para la formación del profesional de la información en Venezuela y Colombia. Códices, 11(2), 65-99.

13. Pirela, Johann; Prieto, César; Pulido, Nelson (2017). Evaluación del perfil profesional en Sistemas de Información, Bibliotecología y Archivística de la Universidad de La Salle, Colombia, desde la perspectiva de los egresados. Rev. cuba. inf. cienc. salud 28(4), 1-18. 